\title{
IL-6-Rezeptorblockade könnte therapeutisch anhaltend wirken
}

Fragestellung: Ist eine anhaltende pharmakologische Blockade des IL-6-Rezeptors bei hoch aktiver Neuromyelitis-opticaSpektrumerkrankung (NMOSE) über längere Zeit wirksam?

Hintergrund: Das proinflammatorische Zytokin IL-6 ist in Serum und Liquor von Patienten mit NMOSE vor allem im akuten Schub erhöht. Einzelfallberichte und eine kleine prospektive Pilotserie schilderten bei hoch aktiver NMOSE Behandlungserfolge mit dem humanisierten monoklonalen IL-6-RezeptorAntikörper Tocilizumab über 12 bis 24 Monate [1].

Patienten und Methodik: In diese multizentrische retrospektive observationale Fallserie wurden acht klinisch und para-

Ringelstein M, Ayzenberg I, Harmel J et al. Long-term therapy with interleukin 6 receptor blockade in highly active neuromyelitis optica spectrum disorder. JAMA Neurol 2015; 72: $756-63$ klinisch gut charakterisierte Frauen mit Aquaporin4-seropositiver, Rituximabrefraktärer NMOSE eingeschlossen. Tocilizumab wurde in einer Dosis von 6-8 $\mathrm{mg} / \mathrm{kg}$ Körpergewicht alle vier bis sechs Wochen intra- venös infundiert. Primärer Endpunkt war die jährliche Schubrate unter Tocilizumab.

Ergebnisse: Im Mittel wurden 29,4 Tocilizumabinfusionen über durchschnittlich 30,9 Monate verabreicht, meist als Monotherapie. Hierunter sank die jährliche Schubrate von im Median vier Schüben vor Therapiebeginn auf 0,4 Schübe unter Tocilizumab ( $\mathrm{p}=0,008)$. Unter intensiverer Therapie mit $8 \mathrm{mg} / \mathrm{kg}$ alle vier Wochen traten tendenziell weniger Schübe auf. Der mediane EDSS-Wert verbesserte sich unter Tocilizumab von 7,3 auf 5,5 $(\mathrm{p}=0,03)$. Sechs Patientinnen hatten vor Therapiebeginn floride MRT-Läsionen, im letzten MRT unter Tocilizumab hingegen nur eine Patientin. Die Aquaporin-4-Antikörpertiter sanken bei sieben von acht Patientinnen $(\mathrm{p}=0,02)$. An Nebenwirkungen wurden erhöhte Cholesterinspiegel in sechs Patientinnen, nicht bedrohliche Infektionen in vier Patientinnen und eine tiefe Beinvenenthrombose und eine Neutropenie in jeweils einer Patientin beobachtet.

Schlussfolgerungen: Tocilizumab könnte eine anhaltend wirksame und sichere Therapieoption bei hoch aktiver NMOSE sein.

\section{- Kommentar von Mathias Buttmann, Würzburg}

\section{Wir brauchen kontrollierte Studien - mit Ihrer Hilfe!}

Diese Fallserie liefert zusammen mit anderen Arbeiten wichtige Hinweise, dass die IL-6-Rezeptorblockade ein wirksames, sicheres und gut verträgliches Therapieprinzip für die hoch aktive NMOSE sein könnte. Tocilizumab ist als RoActemra ${ }^{\circledR}$ zur Behandlung der rheumatoiden Arthritis zugelassen, wo sich in bislang über 50.000 Patientenjahren nach Zulassung ein günstiges Sicherheitsprofil bestätigte [2]. Die Jahresbehandlungskosten liegen bei NMOSE mit etwa $20.000 €$ im Rahmen von Immuntherapien der Multiplen Sklerose. Nicht nur die medizinischen Dienste der Krankenkassen fragen allerdings nach Ergebnissen kontrollierter Studien.

Derzeit wird der Tocilizumabnachfolger SA237 in zwei Phase-3-Studien bei Patienten mit NMOSE untersucht. In Deutschland wird der Antikörper placebokontrolliert in Kombination mit einer immunsuppressiven Behandlung evaluiert. In einer Placebo-kontrollierten Phase-2/3-Studie wird der B-Zelldepletierende Anti-CD19-Antikörper MEDI-551 getestet, der etwas breiter als Rituximab wirkt. Außerdem wird in einer Placebo-kontrollierten Phase-3-Studie der Komplementblockierende Antikörper Eculizumab untersucht, der in einer prospektiven unkontrollierten Studie der Mayo Clinic beeindruckend wirksam war [3]. Alle genannten Studien suchen dringend nach geeigneten Teilnehmern. Wenn Sie einen eventuell studienwilligen Patienten mit aktiver NMOSE betreuen, egal ob mit oder ohne Aquaporin-4-Antikörper, nehmen Sie bitte Kontakt zum nächstgelegenen Zentrum der Neuromyelitis optica Studiengruppe auf (siehe http://www.nemos.net). Nur durch gemeinsame Anstrengung werden wir bei der im Vergleich zur Multiplen Sklerose seltenen NMOSE zu Ergebnissen kontrollierter Studien und damit hoffentlich zugelassenen Medikamenten gelangen.

\footnotetext{
Referenzen:

. Araki M et al. Neurology 2014, 82: $1302-6$

2. Curtis JR et al. Semin Arthritis Rheum 2015, 44: 381 -8

3. Pittock SJ et al. Lancet Neurol 2013, 12: 554-62
}

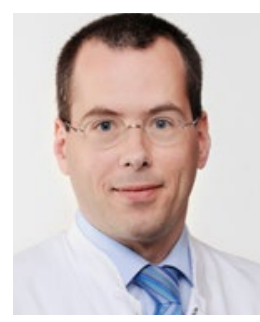

PD Dr. med. Mathias Buttmann, Würzburg

Oberarzt und Leiter der

Neuroimmunologischen Spezialambulanz,

Neurologische Klinik und Poliklinik,

Universitätsklinikum Würzburg

E-Mail:m.buttmann@ukw.de 